\title{
Fabrication of Sericin/Agrose Gel Loaded Lysozyme and Its Potential in Wound Dressing Application
}

\author{
Meirong Yang ${ }^{1}$, Yejing Wang ${ }^{1,2, *}$, Gang Tao ${ }^{1}$, Rui Cai ${ }^{2}$, Peng Wang ${ }^{2}$, Liying Liu ${ }^{1}$, Lisha Ai ${ }^{1}$, \\ Hua Zuo ${ }^{3}$, Ping Zhao ${ }^{1,4}$, Ahmad Umar ${ }^{5}$, Chuanbin Mao ${ }^{6,7}$ and Huawei He ${ }^{1,4, * \text { (i) }}$ \\ 1 State Key Laboratory of Silkworm Genome Biology, Southwest University, Beibei, Chongqing 400715, China; \\ yangmeirong@email.swu.edu.cn (M.Y.); taogang@email.swu.edu.cn (G.T.); \\ 13341345@email.swu.edu.cn (L.L.); als123@email.swu.edu.cn (L.A.); zhaop@swu.edu.cn (P.Z.) \\ 2 College of Biotechnology, Southwest University, Beibei, Chongqing 400715, China; \\ cairui0330@email.swu.edu.cn (R.C.); modelsums@email.swu.edu.cn (P.W.) \\ 3 College of Pharmaceutical Sciences, Southwest University, Beibei, Chongqing 400715, China; \\ zuohua@swu.edu.cn \\ 4 Chongqing Engineering and Technology Research Center for Novel Silk Materials, Southwest University, \\ Beibei, Chongqing 400715, China \\ 5 Department of Chemistry, College of Science and Arts and Promising Centre for Sensors and Electronics \\ Devices (PCSED), Najran University, P.O. Box 1988, Najran 11001, Saudi Arabia; umahmad@nu.edu.sa \\ 6 Department of Chemistry \& Biochemistry, Stephenson Life Science Research Center, \\ University of Oklahoma, 101 Stephenson Parkway, Norman, OK 73019, USA; cbmao@ou.edu \\ 7 School of Materials Science and Engineering, Zhejiang University, Hangzhou 310027, China \\ * Correspondence: yjwang@swu.edu.cn (Y.W.); hehuawei@swu.edu.cn (H.H.); Tel.: +86-23-6825-1575 (H.H.)
}

Received: 6 March 2018; Accepted: 4 April 2018; Published: 13 April 2018

\begin{abstract}
Sericin is a biomaterial resource for its significant biodegradability, biocompatibility, hydrophilicity, and reactivity. Designing a material with superabsorbent, antiseptic, and non-cytotoxic wound dressing properties is advantageous to reduce wound infection and promote wound healing. Herein, we propose an environment-friendly strategy to obtain an interpenetrating polymer network gel through blending sericin and agarose and freeze-drying. The physicochemical characterizations of the sericin/agarose gel including morphology, porosity, swelling behavior, crystallinity, secondary structure, and thermal property were well characterized. Subsequently, the lysozyme loaded sericin/agarose composite gel was successfully prepared by the solution impregnation method. To evaluate the potential of the lysozyme loaded sericin/agarose gel in wound dressing application, we analyzed the lysozyme loading and release, antimicrobial activity, and cytocompatibility of the resulting gel. The results showed the lysozyme loaded composite gel had high porosity, excellent water absorption property, and good antimicrobial activities against Escherichia coli and Staphylococcus aureus. Also, the lysozyme loaded gel showed excellent cytocompatibility on NIH3T3 and HEK293 cells. So, the lysozyme loaded sericin/agarose gel is a potential alternative biomaterial for wound dressing.
\end{abstract}

Keywords: silk sericin; agarose; lysozyme; composite gel; wound dressing

\section{Introduction}

Non-healing skin wounds exposed to bacterial infections are biologically characterized by lengthening inflammation, interfering re-epithelialization, disturbing collagen production, and finally delaying wound healing [1]. In wound care, wound dressing is an important biomedical material used to protect the wound from infection and facilitate wound healing [2]. Accompanied by the growing number of chronic diseases, the wound dressing market is evolving rapidly in the present healthcare 
system worldwide [3]. The ideal wound dressing should absorb wound exudate in a manner, allow gas exchange and maintain necessary moisture at wound interface without cytotoxicity and allergenic response [4]. Besides, it can promote wound healing by creating a suitable microenvironment to prevent bacterial infection and promote cell adhesion and proper proliferation [5]. Among all wound dressing materials, hydrogel is an attractive alternative in traditional therapeutic approaches for its multifunctional abilities such as hydrophilicity, swelling, drug delivery, and in situ gelling capacity [6].

Sericin (SS) is one of the major protein components of silk, which is discarded as a waste during the degumming process in the textile industry [7]. Sericin is a natural protein, exhibiting immense potential in the field of biomaterial owing to its biodegradability, easy availability, and hydrophilicity [8]. Sericin has numerous biological activities such as anti-oxidation, anti-bacterium, and anti-coagulation, promoting cell growth and differentiation [9]. However, sericin is physically fragile and highly soluble due to its amorphous nature [10], which is unsuitable for biomedical applications. Hence, in order to obtain desired material with improved properties for regenerative medicine application, sericin is mostly designed to copolymerize, crosslink, or blend with other polymers as it has polar side chains with diverse functional groups, such as amine, hydroxyl, and carboxyl groups [11-15]. Agarose (AR) is a transparent, neutrally charged, and thermo-reversible natural polysaccharide [16]. Agarose is used extensively in vitro cartilage tissue engineering as it provides a superior foundation for chondrogenesis and higher glycosaminoglycan deposition to produce constructs with functional properties approaching those of native articular cartilage $[17,18]$. Additionally, agarose gel is considered as a biological scaffold material for the central nervous system repair and regeneration due to its excellent mechanical properties which can well match the growth and control of nerve axis, the porous structure which is conducive to nutrient delivery, and the implant which does not cause adverse reactions [19]. However, agarose shows low cell adhesiveness and cell proliferation activity in vivo [20]. Therefore, blending agarose with other polymers such as chitosan and gelatin to overcome the valid drawbacks has escalated in recent years [21,22]. Consequently, the present study brought together the innate advantages of sericin and agarose to fabricate a blended hydrogel for prospective application in a wound dressing.

Nevertheless, the hydrogel is limited as a wound dressing material because it may paradoxically provide a preferred environment for infectious bacteria. To prevent bacterial infection on both skin wound and dressing material, antibiotics such as penicillin and methicillin have been widely used. However, the widespread and indiscriminate use of antibiotics now constitutes a major health concern worldwide due to the emergence of numerous resistant pathogens [23]. Therefore, tremendous attention has been paid to the discovery and development of alternative novel antibiotics, particularly with new modes of action to overcome the resistance. Lysozyme is a natural antibacterial agent that has been isolated from the cells and secretions of virtually all the living organisms [24]. Lysozyme plays the role of the anti-microbial agent through catalyzing the hydrolysis of $\beta-1,4$ glycosidic bonds between $\mathrm{N}$-acetylmuramic acid and $\mathrm{N}$-acetylglucosamine in peptidoglycans of the bacterial cell wall [25]. It is commercially available at low cost, and classified as GRAS grade by the Food and Drug Administration (FDA, US) and as a food additive by the European Union (E 1105) [26]. Lysozyme has been extensively applied as antibacterial agents in wound dressing and protein separation [27]. Accordingly, the development of lysozyme-based antimicrobial material is significantly important toward an environmentally benign antimicrobial field.

We herein developed an improved lysozyme (LZM) loaded sericin/agarose (SS/AR) gel (SS/AR/LZM). Scanning Electronic Microscopy (SEM), Attenuated Total Reflection Fourier Transform Infrared Spectroscopy (ATR-FTIR), X-ray Diffraction (XRD), Thermogravimetric Analysis (TGA), and swelling behavior test were performed to characterize the physicochemical properties of SS/AR gel. We successfully fabricated lysozyme loaded SS/AR composite biomaterials by solution impregnation method. We investigated the lysozyme loading and release, the antimicrobial activity of SS/AR/LZM gel against typical Gram negative/positive bacteria Escherichia coli (E. coli) and Staphyloccus aureus (S. aureus). In addition, the cytotoxicity of the lysozyme loaded SS/AR gel was evaluated on NIH3T3 
and HEK293 cells. The results suggested that the SS/AR/LZM gel with antimicrobial activity and cytocompatibility has a great potential in wound dressing application.

\section{Materials and Methods}

\subsection{Materials}

Bombyx mori cocoons were provided by the State Key Laboratory of Silkworm Genome Biology, Southwest University (China, 400716). Lysozyme (20,000 U/mg) was obtained from Sangon Biotech Co. Ltd. (Shanghai, China). Agarose G-10 was purchased from Biowest (Nuaillé, France). Cell counting kit-8 (CCK-8) was from Beyotime (Beijing, China). LIVE/DEAD cell viability kit was from Thermo Fisher Scientific (Waltham, MA, USA). NIH3T3 (mouse embryonic fibroblast) and HEK293 (human embryonic kidney) cell lines were obtained from China Infrastructure of Cell Line Resources. Chemicals for cell culture such as Dulbecco's modified Eagle's medium (DMEM), Fetal Bovine Serum (FBS), Trypsin-EDTA and Penicillin/Streptomycin were from Gibco BRL (Gaithersburg, MD, USA). Ultrapure water was the product of Milli-Q Plus system from Millipore (Billerica, MA, USA). All other chemicals utilized were of analytical grade.

\subsection{Fabrication of SS/AR Gel}

Sericin was extracted from Bombyx mori cocoons as previous reports [28,29]. Briefly, silkworm cocoons were cut into pieces and autoclaved at $121{ }^{\circ} \mathrm{C}$ for $30 \mathrm{~min}$ to obtain sericin solution. Subsequently, sericin solution was freeze-drying to become sericin powder, and then dissolved in hot water. Sericin and agarose solution $(2 \%, w / v)$ were mixed gently, and allowed to gel after casting into 24-well cell culture plates. These stable hydrogels were then frozen at $-80^{\circ} \mathrm{C}$ for $12 \mathrm{~h}$ followed by lyophilization for $24 \mathrm{~h}$ to become gels. According to the volume ratios of sericin and agarose solution, the corresponding SS / AR gels were termed as S100A0, S75A25, S50A50, S25A75, and S0A100, respectively.

\subsection{Characterization of SS/AR Gel}

SEM observation was performed on JSM-5610LV (Tokyo, Japan) with working voltage of $25 \mathrm{kV}$ to examine the surface morphologies of the gel. The porosities of SS/AR gels were calculated according to the liquid displacement method [30]. Briefly, SS/AR gel was immersed into water (V1) in a graduated cylinder, the total volume including water and SS/AR gel was recorded as V2. The SS/AR gel was then removed from the cylinder and the residual water volume was recorded as V3. The porosity $(p)$ of SS/AR gel was calculated using the following equation:

$$
p=\left(\mathrm{V}_{1}-\mathrm{V}_{3}\right) /\left(\mathrm{V}_{2}-\mathrm{V}_{3}\right) \times 100 \%
$$

ATR-FTIR spectra of sericin and SS/AR gel were determined in the wavenumber range of $650-4000 \mathrm{~cm}^{-1}$ at a resolution of $4 \mathrm{~cm}^{-1}$ on a Nicolet iz10 Infrared spectrophotometer from Thermo Fisher Scientific (Waltham, MA, USA). XRD of sericin and SS/AR gel were carried out by X'Pert powder X-ray diffraction system (PANalytical, Almelo, OV, Netherland) within a $2 \theta$ range of $10^{\circ}-70^{\circ}$. The thermal behaviors of sericin and SS/AR gel were analyzed by a thermogravimetric analyzer TGA-Q50 (TA instruments, New Castle, DE, USA) under a nitrogen flow of $20 \mathrm{~mL} / \mathrm{min}$ [31]. The specimens were heated from room temperature to $600^{\circ} \mathrm{C}$, at a heating rate of $10^{\circ} \mathrm{C} / \mathrm{min}$.

\subsection{Swelling Behavior}

The swelling ability of the SS / AR gel was analyzed using a conventional gravimetric method [32]. Briefly, a pre-weighed dry gel (Wd) was immersed into water at $37^{\circ} \mathrm{C}$ for $30 \mathrm{~min}$ to achieve equilibrium. The swollen weight of gel was recorded as Ws at specific time intervals. The experiment was repeated 
for three times under the same conditions. Swelling ratios (S) were determined as the following equation:

$$
\mathrm{S}=(\mathrm{Ws}-\mathrm{Wd}) / \mathrm{Wd} \times 100 \% .
$$

\subsection{Preparation of SS/AR/LZM Gel}

To prepare the lysozyme loaded SS / AR gel, S50A50 was cut into a circular piece with a diameter of $1.5 \mathrm{~cm}$ and then immersed into lysozyme solution $(20-75 \mathrm{mg} / \mathrm{mL})$ for $16 \mathrm{~h}$. Subsequently, the lysozyme loaded SS/AR gel was removed from lysozyme solution and freeze-dried. According to the lysozyme concentration, the resulting SS/AR/LZM gels were termed as S50A50L20, S50A50L50 and S50A50L75, respectively.

\subsection{The Loading and Release of Lysozyme}

Lysozyme has a specific absorption peak at $280 \mathrm{~nm}$ [33], which could be used to measure the loaded and released lysozyme concentration. Ultraviolet visible spectrophotometer was employed to analyze the loading and release of lysozyme. The loaded lysozyme content was determined by the difference of lysozyme concentration before and after the treatment. The circular SS/AR/LZM gel with a diameter of $1.5 \mathrm{~cm}$ was dispensed into a centrifuge tube containing $4 \mathrm{~mL}$ of $0.01 \mathrm{M}$ PBS (pH 7.4) buffer at $37^{\circ} \mathrm{C}$. At special time points, an aliquot $(1 \mathrm{~mL})$ PBS buffer was collected to measure the absorbance at $280 \mathrm{~nm}$ to determine the released lysozyme contents. Then the gel was transferred into $4 \mathrm{~mL}$ fresh PBS solution for the next measurement. The cumulative release rate was determined according to the ratio of the released and loaded lysozyme. Various lysozyme concentrations $(0.1-0.6 \mathrm{mg} / \mathrm{mL})$ were prepared for the calibration curve. All experiments were performed in triplicate.

\subsection{In Vitro Antibacterial Assay}

The antibacterial activity was evaluated according to the previous procedures with a slight modification [34,35]. SS/AR and SS/AR/LZM gels were cut pieces with $1 \mathrm{~mm}$ in thick and $1.5 \mathrm{~cm}$ in diameter, subsequently sterilized with UV radiation for $30 \mathrm{~min}$. E. coli and S. aureus were grown in Luria-Bertani (LB) media at $37^{\circ} \mathrm{C}$ with continuous shaking until the optical density at $600 \mathrm{~nm}$ $\left(\mathrm{OD}_{600}\right)$ reached about 1.5. Bacteria $(500 \mu \mathrm{L})$ were harvested by centrifugation at $1000 \mathrm{rpm}$ for $5 \mathrm{~min}$ followed by washing with $0.01 \mathrm{M}$ phosphate buffer saline (PBS, pH 7.4). Subsequently, bacteria were re-suspended and diluted with PBS buffer. Next, $50 \mu \mathrm{L}$ of the diluted bacterial suspension was cultured at $37^{\circ} \mathrm{C}$ for $2 \mathrm{~h}$ in the presence of SS/AR gel or SS/AR/LZM gel. Aliquots $(1 \mu \mathrm{L})$ of the mixture were diluted $(1: 10,000)$ in PBS, and then manually spread on LB agar plates. After $16 \mathrm{~h}$ incubation at $37^{\circ} \mathrm{C}$, the units of colony formation in each agar plate were counted to check the antibacterial ability of the gels. Each independent experiment was performed in triplicate.

\subsection{Cytocompatibility Assay}

NIH3T3 and HEK293 cells were cultured in high glucose DMEM supplemented with $10 \%$ FBS and $1 \%$ penicillin/streptomycin in a humidified atmosphere of $95 \%$ and $5 \% \mathrm{CO}_{2}$ at $37^{\circ} \mathrm{C}$. To check the cell viability, NIH3T3 and HEK293 cells $(100 \mu \mathrm{L})$ were loaded at the density of $1 \times 10^{4}$ cells $/$ well in 96-well plates and incubated $12 \mathrm{~h}$ at $37^{\circ} \mathrm{C}$. SS/AR or SS/AR/LZM gel was sterilized by ultraviolet radiation overnight and then added to the cell plates. Non-treated cells were used as a control.

After treated with the gels for $12 \mathrm{~h}, 24 \mathrm{~h}$ and $36 \mathrm{~h}, \mathrm{CCK}-8$ assay was used to assess cell viability according to the manufacturer's instructions. CCK-8 solution $(10 \mu \mathrm{L})$ was added into each well and then incubated at $37^{\circ} \mathrm{C}$ for $1.5 \mathrm{~h}$. The optical density (OD) of each well was measured at $450 \mathrm{~nm}$ on a microplate reader TECAN (Mannedorf, Switzerland). The cell viability is defined as the percentage of OD value of the treated and control wells. For each experiment, at least three samples were evaluated. The morphologies of NIH3T3 and HEK293 cells after incubation for $24 \mathrm{~h}$ in the absence and presence of SS/AR or SS/AR/LZM gel were observed on a fluorescence microscope EVOS FL Auto Cell Imaging System (Life, Bothell, WA, USA). 
Additionally, LIVE/DEAD staining assay was carried out to further assess the effects of the gels on the cells viability. NIH3T3 and HEK293 cells were cultured and incubated at $37{ }^{\circ} \mathrm{C}$ as described above. The cells after treated with SS/AR or SS/AR/LZM gel for $24 \mathrm{~h}$ were incubated with staining solution at $37^{\circ} \mathrm{C}$ for $15 \mathrm{~min}$. Then the images were collected on EVOS FL Auto Cell Imaging System. For each sample, the experiment was done in triplicate.

\section{Results and Discussion}

\subsection{Preparation of SS/AR/LZM Gel}

In this study, we prepared the SS/AR/LZM gel with good antibacterial activity and cytocompatibility, as illustrated in Figure 1. Sericin and agarose solution were mixed and then freeze-dried to become a porous gel. Thereafter, lysozyme, a natural antimicrobial agent, was loaded into the SS/AR gel. As sericin is negatively charged, agarose is neutral, whereas lysozyme is positively charged, thus the adsorption of lysozyme into the SS/AR gel may be attributed to the electrostatic interactions between the opposite charges of sericin and lysozyme [36]. Also, the physical adsorption caused by free diffusion could promote the adsorption of lysozyme. In addition, lysozyme has carboxyl group, amino groups and four disulfide bonds [37], and sericin has hydroxyl, carboxyl, and amino groups [38]. The special hydrophilic/hydrophobic interactions between lysozyme and sericin are also able to enhance the adsorption of lysozyme. The resulting SS/AR/LZM gel with interconnected porous structures, high swelling ability, good antibacterial activity and cytocompatibility may be a prospective alternative for wound dressing.

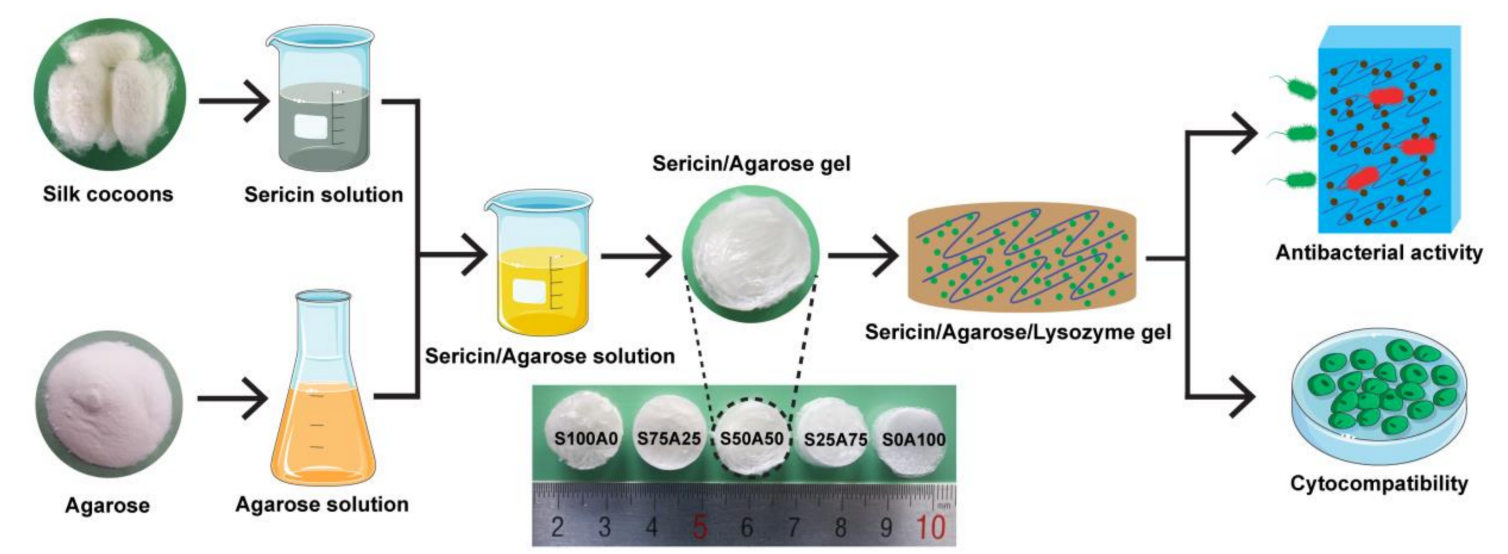

Figure 1. Schematic illustration of the fabrication of sericin (SS)/agarose (AR)/lysozyme (LZM) gel.

\subsection{Morphology of SS/AR Gel}

Porous materials provide space for cell growth and proliferation, and the microenvironment for the retention and release of bioactive molecules [39]. Furthermore, the porous structure affects the supply of nutrients and oxygen, and the removal of wastes [40], which is of utmost importance to wound dressing. In this study, the prepared gels had macro-porous "open-cell" structures (Figure 2A-D). The porosity of S75A25, S50A50, S25A75, and S0A100 gels were 53.17\%, 49.54\%, 46.17\% and 59.75\%, respectively (Figure 2E). Compared to other gels, S50A50 and S25A75 gels had significantly bigger pore sizes. This may be the fact that S50A50 and S25A75 gels could adsorb more water. After lyophilization, the space water occupied resulted in the formation of pores. Consequently, the pore size and porosity of gels were dependent on the ratio of sericin and agarose solution. 

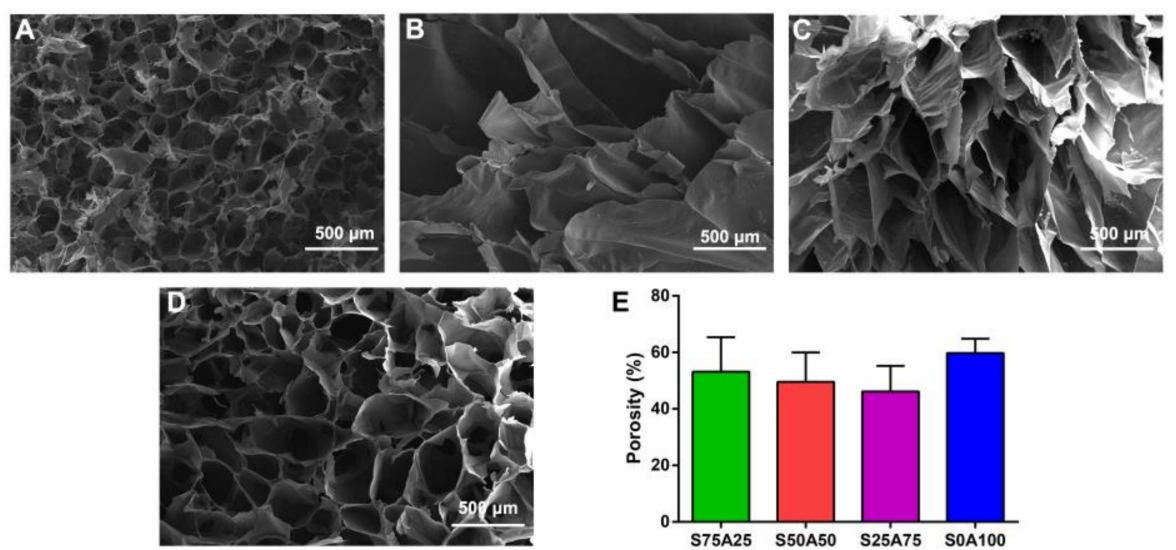

Figure 2. The porous microstructures of S75A25 (A), S50A50 (B), S25A75 (C), and S0A100 (D) gels. The porosity of gels with different ratios of sericin and agarose (E).

\subsection{Characterization of SS/AR Gel}

ATR-FTIR was employed to analyze the chemical interactions between sericin and agarose as ATR technique can probe to only a shallow depth and thus emphasize any surface coatings [41]. Sericin has a typical spectrum with distinctive peaks at $1600-1700 \mathrm{~cm}^{-1}$ (amide I, C=O stretching vibration), $1480-1575 \mathrm{~cm}^{-1}$ (amide II, N-H bending vibration), and 1229-1301 $\mathrm{cm}^{-1}$ (amide III) [42]. As shown in Figure 3A, sericin gel had characteristic peaks at $1621 \mathrm{~cm}^{-1}, 1521 \mathrm{~cm}^{-1}$, and $1241 \mathrm{~cm}^{-1}$, corresponding to amide I, amide II, and amide III, respectively. Pure agarose exhibited its characteristic peaks at $1068 \mathrm{~cm}^{-1}$ (C-O, axial deformation), $930 \mathrm{~cm}^{-1}$ (3, 6-anhydro-galactose), and $891 \mathrm{~cm}^{-1}$ (C-H, angular deformation of $\beta$ anomeric carbon), the result was consistent with the previous study [43]. In the blended gels, the characteristic peaks of both agarose and sericin were recorded, which confirmed the presence of both components. Some slight shifts in amide I and amide II peaks, and the differences in the intensity of peaks were observed in case of the composite gels, which indicated that the backbone structures of sericin and agarose did not change. Lysozyme has characteristic peaks at $3295 \mathrm{~cm}^{-1}$ (NAH stretching of the free amino groups), and $2961 \mathrm{~cm}^{-1}$ (CAH stretching) as well7 as amide I (1600-1700 $\left.\mathrm{cm}^{-1}\right)$, amide II $\left(1500-1600 \mathrm{~cm}^{-1}\right)$ and amide III $\left(1230-1320 \mathrm{~cm}^{-1}\right)$ [44]. Few peaks were found to overlap with the peaks of sericin.

The crystalline structure of the composite was analyzed by XRD. Silk protein has main diffraction peaks of Silk I $\left(2 \theta=12.2^{\circ}\right.$ and $\left.28.2^{\circ}\right)$, and Silk II $\left(2 \theta=18.9^{\circ}\right.$ and $\left.20.7^{\circ}\right)$ [45]. Similar XRD patterns of sericin with peaks at $2 \theta=19.2^{\circ}$ and $21.15^{\circ}$ have been reported [46-49]. Sericin and SS/AR gel exhibited obvious diffraction peaks at $19.08^{\circ}$ and $19.56^{\circ}$, respectively (Figure $3 \mathrm{~B}$ ), which indicated the existence of sericin in the SS/AR gel. The difference of $2 \theta$ between sericin and SS/AR gel reflect the conversion of the random coil to $\beta$-sheet structure for the presence of the intermolecular hydrogen bond in sericin [50].

The thermal stability of sericin and SS/AR gel were examined by TGA. Sericin and SS/AR gel underwent three stages of thermal degradation including dehydration, deploymerization, and decomposition (Figure 3C). The first stage was from room temperature to around $110{ }^{\circ} \mathrm{C}$, where the mass loss revealed the removal of adsorbed water molecules in sericin and SS/AR gel. The second major decomposition was attributed to the degradation of sericin and agarose occurred in the temperature range of $120-410^{\circ} \mathrm{C}$. At this stage, the mass loss of SS/AR gel was faster than that of sericin, indicating that sericin could improve the thermal stability of the composite gels and delay the thermal degradation process. At the last stage, the mass loss occurred from $420{ }^{\circ} \mathrm{C}$ to $600{ }^{\circ} \mathrm{C}$, which was associated with the breakdown of sericin and agarose. 
A
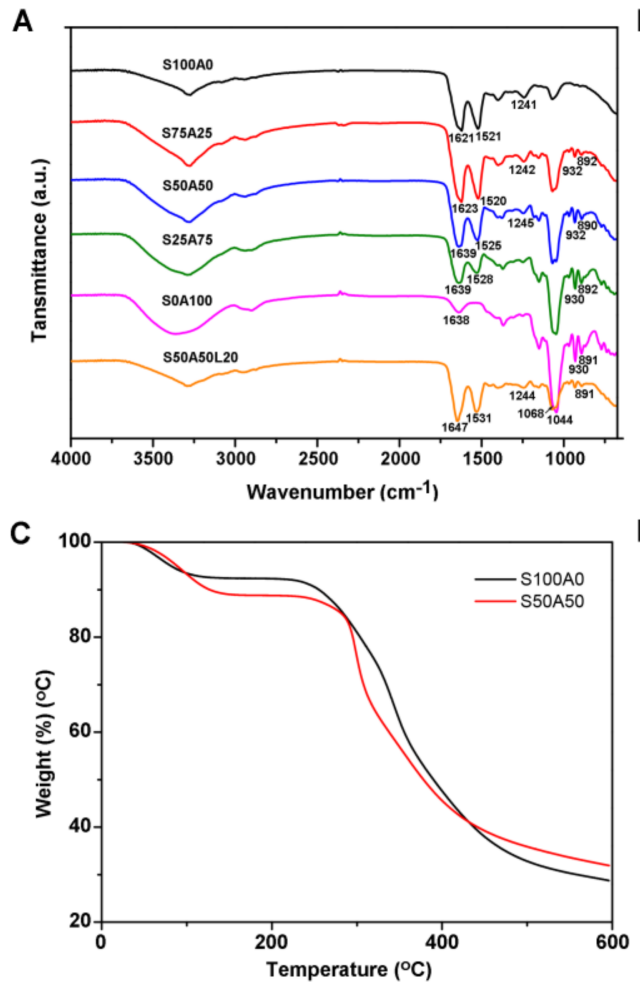

B
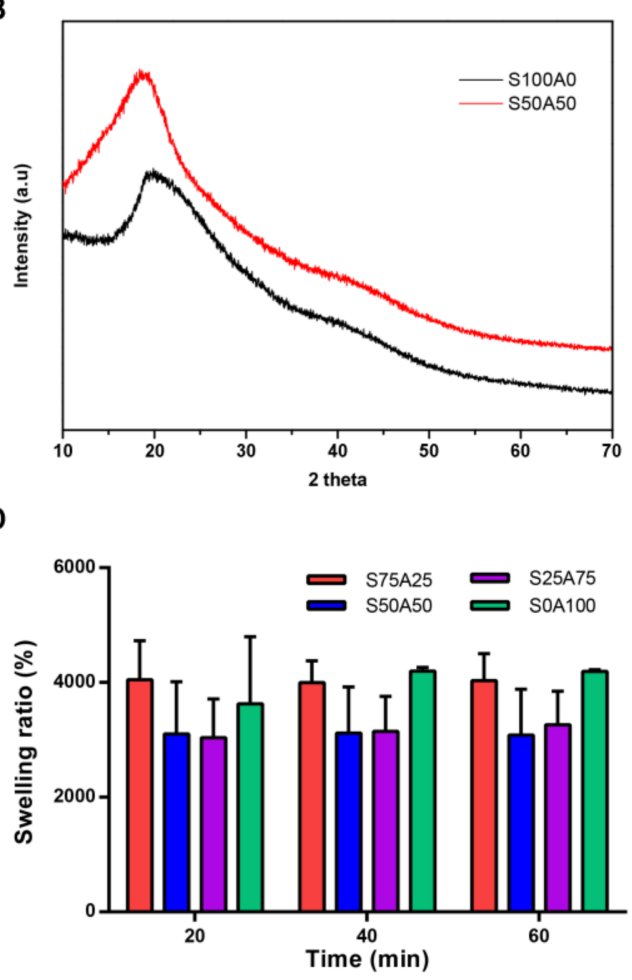

Figure 3. Characterizations of SS/AR gels. (A) Attenuated Total Reflection Fourier Transform Infrared Spectroscopy (ATR-FTIR); (B) X-Ray Diffraction (XRD); (C) Thermogravimetric Analysis (TGA); and (D) swelling ratio.

\subsection{Swelling Behavior}

Swelling ratio is a vital property to illustrate the uptake of liquids in wound dressing. The swelling ratio of various SS/AR gels were shown in Figure 3D. The results showed that all samples exhibited good swelling behavior. After $60 \mathrm{~min}, \mathrm{~S} 75 \mathrm{~A} 25$ and S0A100 gels had the swelling ratios of 3628-4196\%, whereas S50A50 and S25A75 had the swelling ratios of 3040-3262\%. The swelling ratios of S75A25 and S0A100 gels were higher than those of S50A50 and S25A75 as they had smaller pore size and higher porosities. The swelling of the gels had two stages, including the growing period and equilibrium period. In the initial $20 \mathrm{~min}$, the swelling ratios of all samples increased quickly, indicating the excellent hydrophilicity of the composite gels. Thereafter, all gels quickly reached the swelling equilibrium.

The SS / AR gels with various ratios had a honeycomb structure, high porosity as well as excellent swelling capacity. In this study, we purposed to develop an alternative wound dressing through bringing together the innate advantages of sericin and agarose. High content of sericin will reduce the mechanical property of the gel, and high content of agarose may affect the cell adhesion and proliferation. Hence, we suggested the SS/AR gel with a ratio of 50:50 (S50A50) had moderate mechanical property and cytocompatibility, which may be suitable for wound dressing. The S50A50 gel was chosen for the further experiments.

\subsection{Lysozyme Release}

To avoid frequent replacement of the dressing and reduce the risk of overexposing wound to bacteria, a wound dressing should have a controllable drug release ability [51,52]. The loaded lysozyme contents of S50A50L20 and S50A50L50 gels were $24.94 \mathrm{mg}$ and $50.78 \mathrm{mg}$, respectively (Figure 4A). And the loading efficiency of lysozyme was $62 \%$ and 51\% for S50A50L20 and S50A50L50 gels, respectively (Figure 4B). Increasing lysozyme solution concentration could increase the loaded lysozyme contents, however, reduce its loading efficiency. To assess lysozyme release, we analyzed the 
correlation between lysozyme concentration and UV absorption by the standard curve. The results showed that UV absorption was tightly correlated with lysozyme concentration (Figure 4C). Obviously, lysozyme could be released from both S50A50L20 and S50A50L50 gels. Lysozyme release could be divided into burst stage and steady stage (Figure 4D). During the initial burst phase, the release rate was relatively high. This effect is associated with the diffusion of water molecules and desorption of lysozyme close to the surface of the gel [53]. At the steady phase, lysozyme was gradually released from SS/AR/LZM gels up to $60 \mathrm{~h}$. The cumulative release of S50A50L20 and S50A50L50 gels reached $74 \%$ and $86 \%$ at $3 \mathrm{~h}$, respectively. After $60 \mathrm{~h}$, the cumulative release reached $98 \%$ and $99 \%$, indicating lysozyme was almost entirely released from the composite gels. The results suggested that the SS/AR/LZM gel had a sustainable lysozyme releasing ability, which is required for a wound dressing.

A

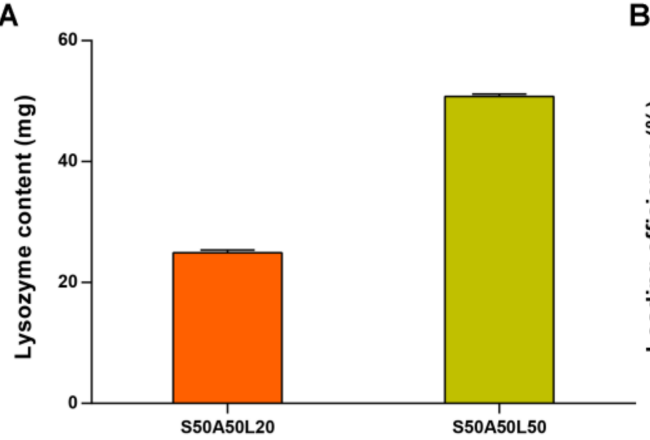

\section{C}

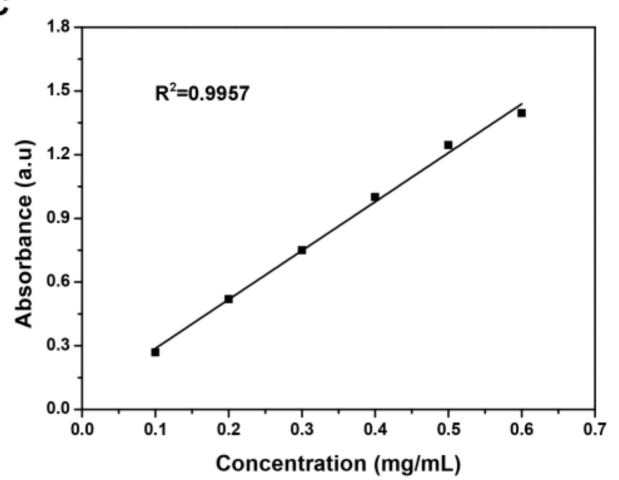

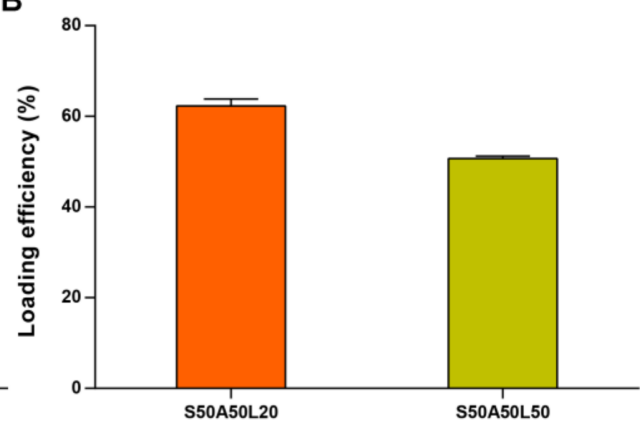

D

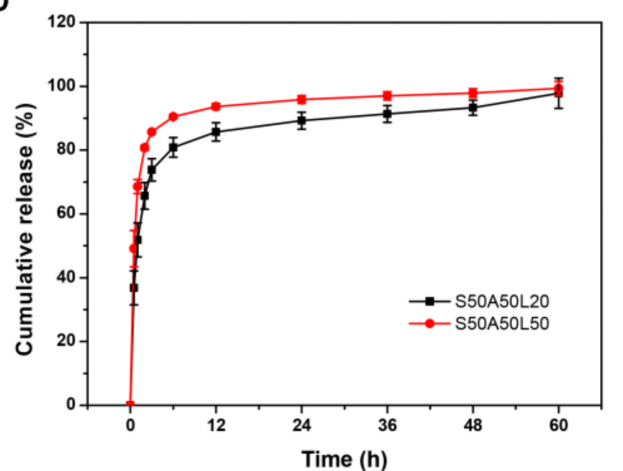

Figure 4. The loading and release of lysozyme. (A) Lysozyme contents loaded on SS/AR gel; (B) loading efficiency; (C) standard curves of UV intensity and lysozyme concentration; and (D) the cumulative release of lysozyme.

\subsection{Antibacterial Activity}

The antibacterial activity of the composite gels toward E. coli and S. aureus were shown in Figure 5A. Compared to the control, the total colonies number in the presence of SS/AR/LZM gels significantly decreased, indicating the SS / AR/LZM gel had good antibacterial activity. The S50A50L20 and S50A50L50 gels exhibited the bacteria reduction rates of $76 \% / 87 \%$ and $84 \% / 95 \%$ toward E. coli, S. aureus, respectively. The S50A50L75 gel completely inhibited the growth of the bacteria both for E. coli and S. aureus, suggesting increasing lysozyme solution concentration can improve the bactericidal ability of the composite gel as it increased the loaded and released lysozyme contents. 
A
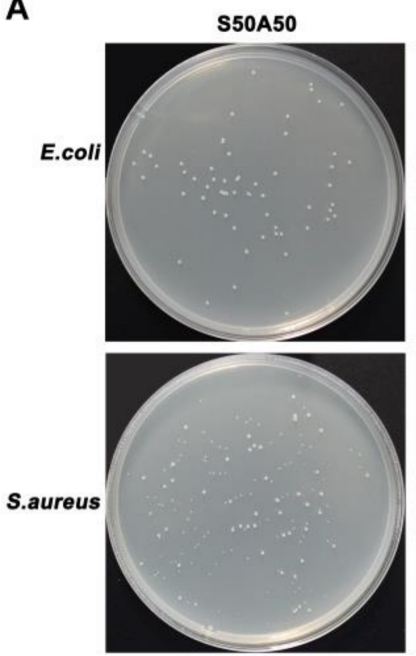

B

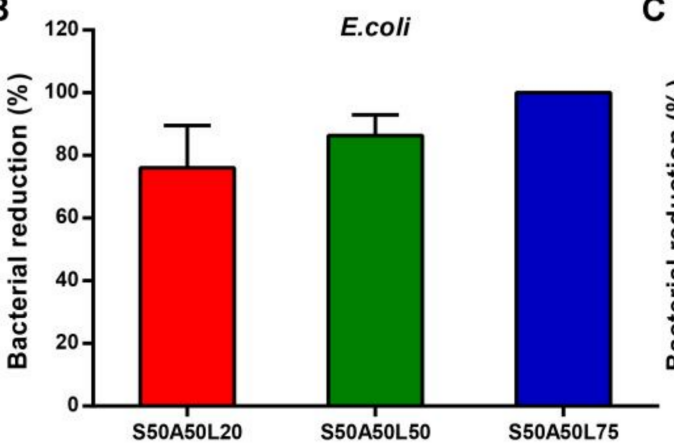

S50A50L20
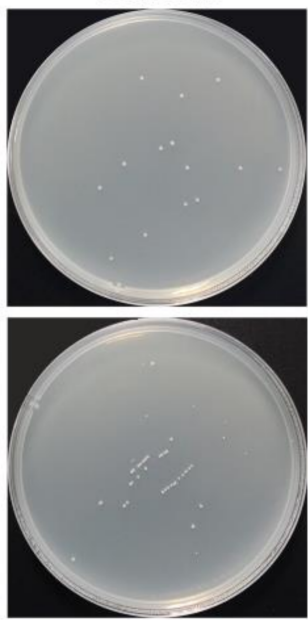

C
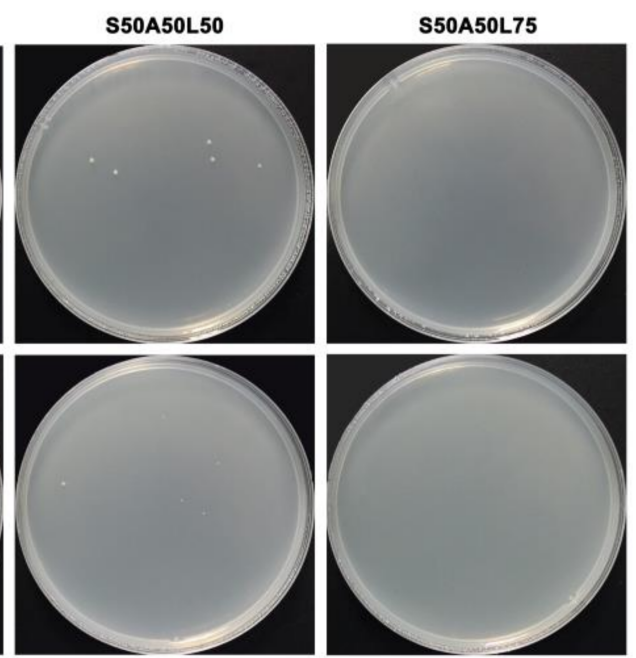

S.aureus

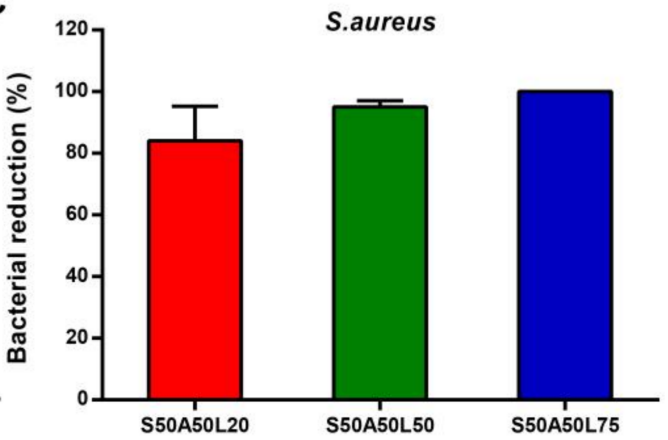

Figure 5. Antibacterial activities of SS/AR/LZM gels against E. coli and S. aureus. (A) Total bacterial colonies counting; (B, C) Bacterial colonies reduction rate.

Lysozyme is a natural antibacterial agent. Its antibacterial activity is mild compared with inorganic and organic antibacterial agents. To improve the antibacterial effect of lysozyme, some strategies such as physical and chemical modifications or synergistic action with other substances have been developed in recent years [26,54-56]. In this study, the SS/AR/LZM gels for antibacterial test had a dimension of $1 \mathrm{~mm}$ in thickness and $1.5 \mathrm{~cm}$ in diameter. The size and thickness determined the low loading content of lysozyme. In addition, the bacterial suspension volume affected the release efficiency of lysozyme from the gels. Therefore, SS / AR/LZM gels exhibited expected antibacterial effect; however, the loading content and the release efficiency of lysozyme determined its antibacterial activity.

Various antimicrobial materials have been developed for wound dressing application, such as metals/metal oxides [57], antibiotics [58], and peptides [2]. These materials are associated with a big concern about adverse effects on ecosystems. For instance, silver nanoparticle, one of the most classical and important materials, exert an adverse effect on environmental safety during its preparation and application. Also, the widespread and indiscriminate use of antibiotics response to the emergence of numerous resistant pathogens. The cost of the synthetic antibacterial peptide is very high. Here, the developed SS/AR/LZM material has good cytocompatibility and without toxic side effects and drug resistance. The main advantage of the lysozyme-based material is significantly safe to human and environment, which has exciting and expected potentials in biomedical materials such as wound dressing.

\subsection{Cytocompatibility Assay}

To evaluate the cytocompatibility of SS/AR and SS/AR/LZM gels, we tested the effects of SS/AR and SS/AR/LZM gels on the viability of NIH3T3 and HEK293 cells. In the CCK-8 test, a soluble 
formazan dye with the maximum absorbance at $450 \mathrm{~nm}$ produced as metabolically active cells react with a tetrazolium salt in the CCK-8 reagent. And, the higher optical density (OD) value indicates better cell viability and more live cells [59]. As shown in Figure 6A, B, after $12 \mathrm{~h}$, there were no significant differences in the cells viabilities among SS/AR, SS/AR/LZM gels and the control. After $24 \mathrm{~h}$, the cells viabilities for SS/AR, SS/AR/LZM, and control groups increased quickly. After $36 \mathrm{~h}$, the cells in all groups exhibited significantly higher viabilities than those at $24 \mathrm{~h}$. The results suggested the SS/AR/LZM gel had good cytocompatibility on NIH3T3 and HEK293 cells. This may be due to the fact that active sericin promotes cell growth for its cytoprotective and mitogenic abilities [9]. Furthermore, the morphologies of NIH3T3 and HEK293 cells were observed on an optical microscope after $24 \mathrm{~h}$ in the absence and presence of SS/AR and SS/AR/LZM gels. The results showed that morphologies of the cells were nearly identical to that of the control (Figure 6C), indicating SS/AR and SS / AR/LZM gels were not toxic on NIH3T3 and HEK293 cells.

After staining with the LIVE/DEAD fluorescent reagent, the alive cells were stained green while the apoptotic cells were stained red. The result showed most of the cells were green and only a few of cells were red (Figure 7), indicating that the SS / AR and SS / AR/LZM gels had good cytocompatibility on NIH3T3 and HEK293 cells. The fluorescence images of the LIVE/DEAD staining assay were in accordance with the results of CCK-8 assay.

A

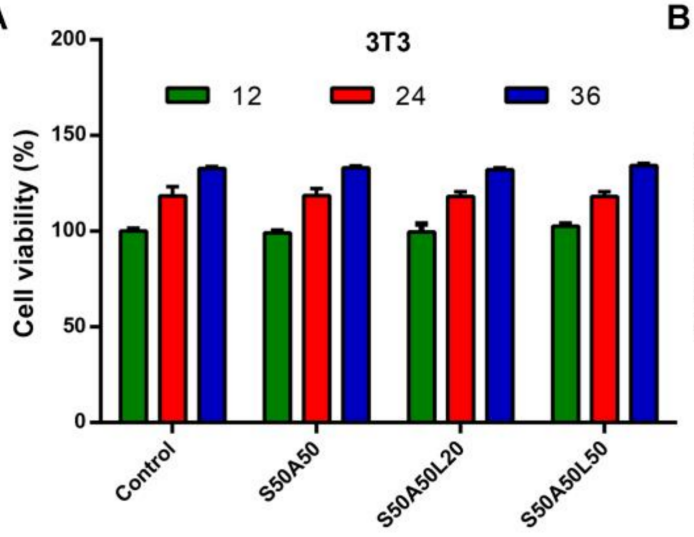

C

Control
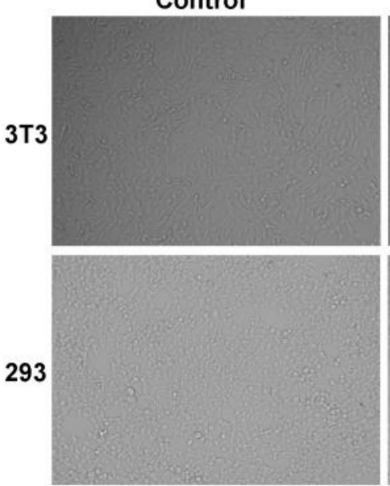

S50A50
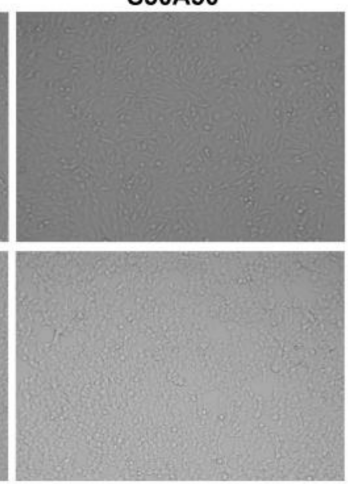

B

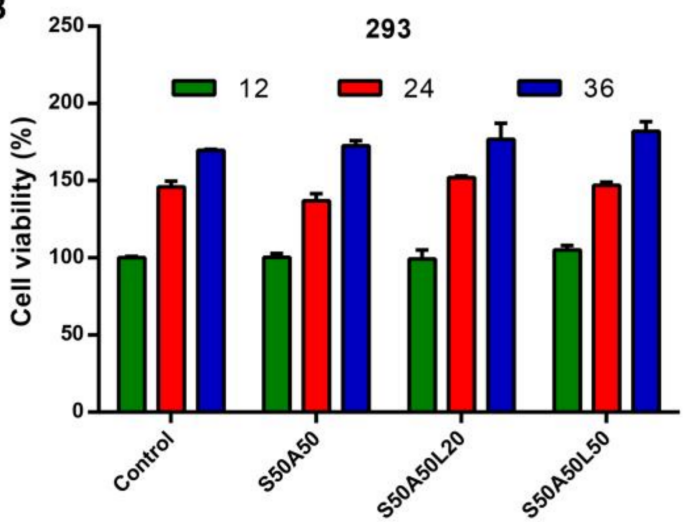

S50A50L20

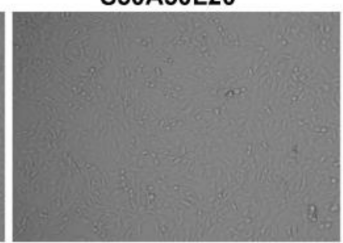

S50A50L50

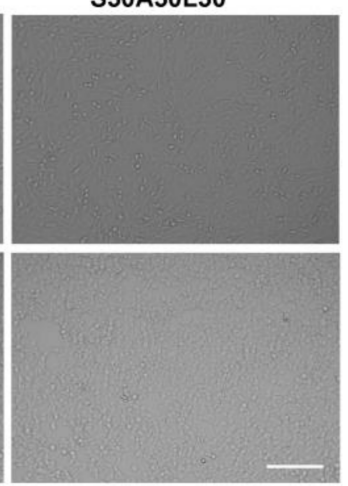

Figure 6. CCK-8 assay of the SS/AR/LZM gels. Cells viability of NIH3T3 (A) and HEK293 (B) in the presence of SS/AR gel or SS / AR/LZM gel, respectively. Microscopic analysis of NIH3T3 and HEK293 cells; ((C), scale bar, $200 \mu \mathrm{m})$. 
A
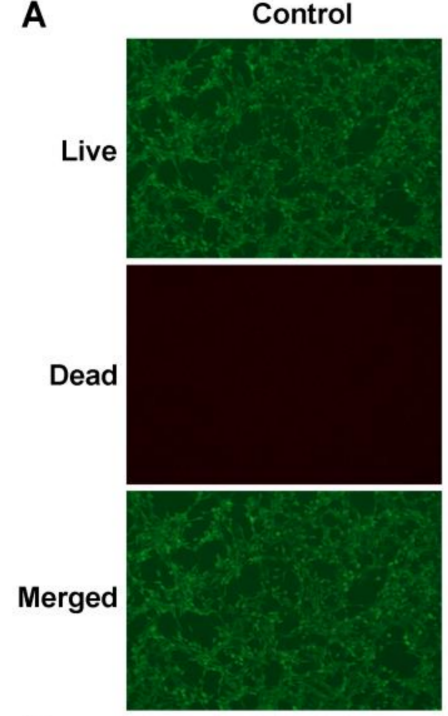

B
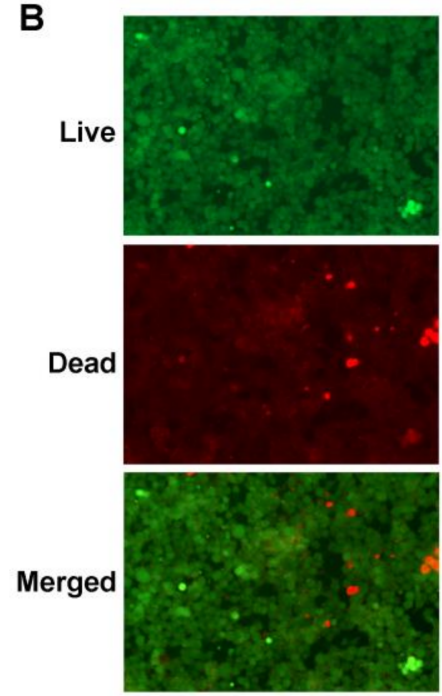

S50A50
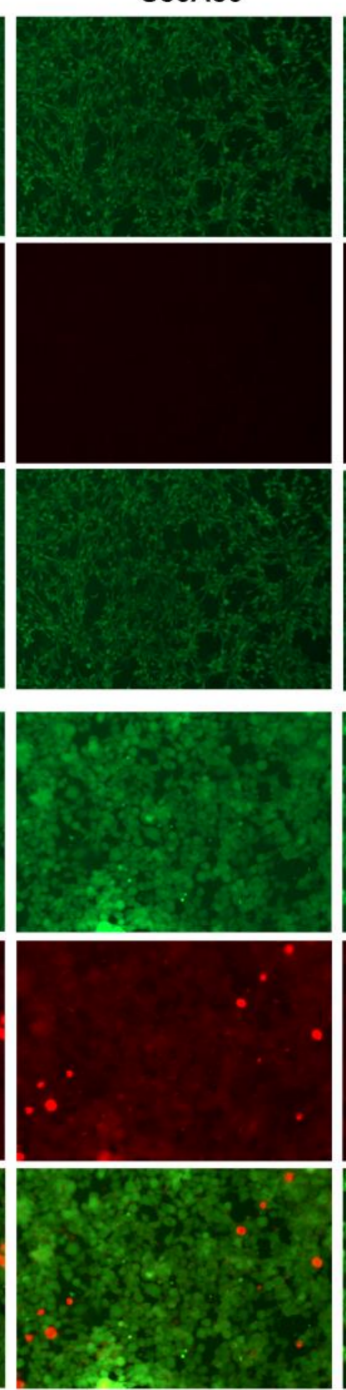

S50A50L20
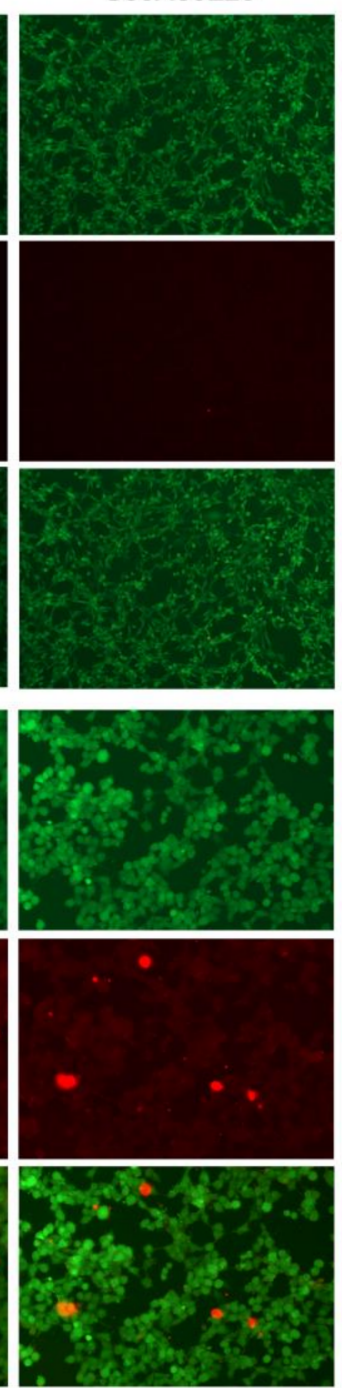

S50A50L50
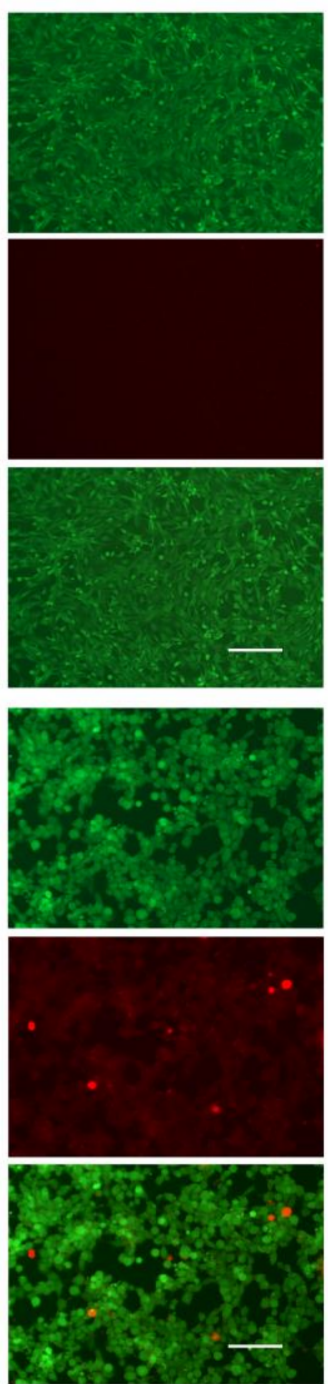

Figure 7. The LIVE/DEAD staining assay. NIH3T3 ((A), scale bar, $200 \mu \mathrm{m})$ and HEK293 ((B), scale bar, $100 \mu \mathrm{m})$ cells.

\section{Conclusions}

In summary, the SS/AR composite gel was successfully prepared by blending and freeze-drying. The SS/AR gel had highly porous and inter-connected structure, and good swelling behavior. The lysozyme loaded SS/AR gel had a sustainable lysozyme releasing ability and good antimicrobial activities against E. coli and S. aureus as well as excellent cytocompatibility on NIH3T3 and HEK293 cells. The SS/AR/LZM gel is expected to develop as an alternative for wound dressing.

Acknowledgments: This work was supported by the National Natural Science Foundation of China (31572465, 51673168), the State Key Program of National Natural Science of China (31530071), Chongqing Research Program of Basic Research and Frontier Technology (cstc2015jcyja00040, cstc2015jcyjbx0035), the Fundamental Research Funds for the Central Universities (XDJK2018B010, XDJK2018C063), the Graduate Research and Innovation Project of Chongqing (CYS17076, CYS17081, CYB17069), Open Project Program of Chongqing Engineering and Technology Research Center for Novel Silk Materials (silkgczx2016003), and the National Key Research and Development Program of China (2016YFA0100900).

Author Contributions: Meirong Yang, Yejing Wang and Huawei He conceived and designed the experiments; Meirong Yang, Peng Wang, Liying Liu, and Lisha Ai performed the experiments; Meirong Yang and Gang Tao analyzed the data; Gang Tao, Rui Cai, and Hua Zuo contributed reagents/materials/analysis tools; Meirong Yang, 
Huawei He, Yejing Wang, Ping Zhao wrote the paper; Meirong Yang, Huawei He, Umar Ahmad and Chuanbin Mao revised the paper.

Conflicts of Interest: The authors declare no conflict of interest.

\section{References}

1. Edwards, R.; Harding, K.G. Bacteria and wound healing. Curr. Opin. Infect. Dis. 2004, 17, 91-96. [CrossRef] [PubMed]

2. Song, D.W.; Kim, S.H.; Kim, H.H.; Lee, K.H.; Ki, C.S.; Park, Y.H. Multi-biofunction of antimicrobial peptide-immobilized silk fibroin nanofiber membrane: Implications for wound healing. Acta Biomater. 2016, 39, 146-155. [CrossRef] [PubMed]

3. Sheikh, E.S.; Sheikh, E.S.; Fetterolf, D.E. Use of dehydrated human amniotic membrane allografts to promote healing in patients with refractory non healing wounds. Int. Wound J. 2014, 11, 711-717. [CrossRef] [PubMed]

4. Boateng, J.; Catanzano, O. Advanced therapeutic dressings for effective wound healing. J. Pharm. Sci. 2015, 104, 3653-3680. [CrossRef] [PubMed]

5. Stumpf, T.R.; Pertile, R.A.; Rambo, C.R.; Porto, L.M. Enriched glucose and dextrin mannitol-based media modulates fibroblast behavior on bacterial cellulose membranes. Mater. Sci. Eng. C 2013, 33, 4739-4745. [CrossRef] [PubMed]

6. Xie, Z.; Aphale, N.V.; Kadapure, T.D.; Wadajkar, A.S.; Orr, S.; Gyawali, D.; Qian, G.; Nguyen, K.T.; Yang, J. Design of antimicrobial peptides conjugated biodegradable citric acid derived hydrogels for wound healing. J. Biomed. Mater. Res. A 2015, 103, 3907-3918. [CrossRef] [PubMed]

7. Zhang, Y.-Q. Applications of natural silk protein sericin in biomaterials. Biotechnol. Adv. 2002, 20, 91-100. [CrossRef]

8. Kundu, S.C.; Dash, B.C.; Dash, R.; Kaplan, D.L. Natural protective glue protein, sericin bioengineered by silkworms: Potential for biomedical and biotechnological applications. Prog. Polym. Sci. 2008, 33, 998-1012. [CrossRef]

9. Lamboni, L.; Gauthier, M.; Yang, G.; Wang, Q. Silk sericin: A versatile material for tissue engineering and drug delivery. Biotechnol. Adv. 2015, 33, 1855-1867. [CrossRef] [PubMed]

10. Wang, Z.; Zhang, Y.; Zhang, J.; Huang, L.; Liu, J.; Li, Y.; Zhang, G.; Kundu, S.C.; Wang, L. Exploring natural silk protein sericin for regenerative medicine: An injectable, photoluminescent, cell-adhesive 3D hydrogel. Sci. Rep. 2014, 4, 7064. [CrossRef] [PubMed]

11. Kundu, B.; Kundu, S.C. Silk sericin/polyacrylamide in situ forming hydrogels for dermal reconstruction. Biomaterials 2012, 33, 7456-7467. [CrossRef] [PubMed]

12. Cho, K.Y.; Moon, J.Y.; Lee, Y.W.; Lee, K.G.; Yeo, J.H.; Kweon, H.Y.; Kim, K.H.; Cho, C.S. Preparation of self-assembled silk sericin nanoparticles. Int. J. Biol. Macromol. 2003, 32, 36-42. [CrossRef]

13. Nayak, S.; Talukdar, S.; Kundu, S.C. Potential of 2D crosslinked sericin membranes with improved biostability for skin tissue engineering. Cell Tissue Res. 2012, 347, 783-794. [CrossRef] [PubMed]

14. Aramwit, P.; Siritientong, T.; Kanokpanont, S.; Srichana, T. Formulation and characterization of silk sericin-PVA scaffold crosslinked with genipin. Int. J. Biol. Macromol. 2010, 47, 668-675. [CrossRef] [PubMed]

15. He, H.; Cai, R.; Wang, Y.; Tao, G.; Guo, P.; Zuo, H.; Chen, L.; Liu, X.; Zhao, P.; Xia, Q. Preparation and characterization of silk sericin/PVA blend film with silver nanoparticles for potential antimicrobial application. Int. J. Biol. Macromol. 2017, 104, 457-464. [CrossRef] [PubMed]

16. Bellamkonda, R.; Ranieri, J.P.; Bouche, N.; Aebischer, P. Hydrogel-based three-dimensional matrix for neural cells. J. Biomed. Mater. Res. 1995, 29, 663-671. [CrossRef] [PubMed]

17. Yodmuang, S.; McNamara, S.L.; Nover, A.B.; Mandal, B.B.; Agarwal, M.; Kelly, T.A.; Chao, P.H.; Hung, C.; Kaplan, D.L.; Vunjak-Novakovic, G. Silk microfiber-reinforced silk hydrogel composites for functional cartilage tissue repair. Acta Biomater. 2015, 11, 27-36. [CrossRef] [PubMed]

18. Lima, E.G.; Bian, L.; Ng, K.W.; Mauck, R.L.; Byers, B.A.; Tuan, R.S.; Ateshian, G.A.; Hung, C.T. The beneficial effect of delayed compressive loading on tissue-engineered cartilage constructs cultured with TGF- $\beta 3$. Osteoarthr. Cartil. 2007, 15, 1025-1033. [CrossRef] [PubMed]

19. Stokols, S.; Tuszynski, M.H. Freeze-dried agarose scaffolds with uniaxial channels stimulate and guide linear axonal growth following spinal cord injury. Biomaterials 2006, 27, 443-451. [CrossRef] [PubMed] 
20. Gruber, H.E.; Fisher, E.C., Jr.; Desai, B.; Stasky, A.A.; Hoelscher, G.; Hanley, E.N., Jr. Human intervertebral disc cells from the annulus: Three-dimensional culture in agarose or alginate and responsiveness to TGF- $\beta 1$. Exp. Cell Res. 1997, 235, 13-21. [CrossRef] [PubMed]

21. Doulabi, A.; Mequanint, K.; Mohammadi, H. Blends and nanocomposite biomaterials for articular cartilage tissue engineering. Materials 2014, 7, 5327-5355. [CrossRef] [PubMed]

22. Bhat, S.; Tripathi, A.; Kumar, A. Supermacroprous chitosan-agarose-gelatin cryogels: In vitro characterization and in vivo assessment for cartilage tissue engineering. J. R. Soc. Interfaces 2011, 8, 540-554. [CrossRef] [PubMed]

23. Nikaido, H. Multidrug resistance in bacteria. Annu. Rev. Biochem. 2009, 78, 119-146. [CrossRef] [PubMed]

24. Benkerroum, N. Antimicrobial activity of lysozyme with special relevance to milk. Afr. J. Biotechnol. 2008, 7, 4856-4867.

25. Hamdani, A.M.; Wani, I.A.; Bhat, N.A.; Siddiqi, R.A. Effect of guar gum conjugation on functional, antioxidant and antimicrobial activity of egg white lysozyme. Food Chem. 2018, 240, 1201-1209. [CrossRef] [PubMed]

26. Gu, J.; Su, Y.; Liu, P.; Li, P.; Yang, P. An environmentally benign antimicrobial coating based on a protein supramolecular assembly. ACS Appl. Mater. Interfaces 2017, 9, 198-210. [CrossRef] [PubMed]

27. Zhao, J.; Wang, X.; Kuang, Y.; Zhang, Y.; Shi, X.; Liu, X.; Deng, H. Multilayer composite beads constructed via layer-by-layer self-assembly for lysozyme controlled release. RSC Adv. 2014, 4, 24369-24376. [CrossRef]

28. Yang, M.; Wang, Y.; Cai, R.; Tao, G.; Chang, H.; Ding, C.; Zuo, H.; Shen, H.; Zhao, P.; He, H. Preparation and characterization of silk sericin/glycerol films coated with silver nanoparticles for antibacterial application. Sci. Adv. Mater. 2018, 10, 1-8. [CrossRef]

29. He, H.; Tao, G.; Wang, Y.; Cai, R.; Guo, P.; Chen, L.; Zuo, H.; Zhao, P.; Xia, Q. In situ green synthesis and characterization of sericin-silver nanoparticle composite with effective antibacterial activity and good biocompatibility. Mater. Sci. Eng. C 2017, 80, 509-516. [CrossRef] [PubMed]

30. Nazarov, R.; Jin, H.J.; Kaplan, D.L. Porous 3-D scaffolds from regenerated silk fibroin. Biomacromolecules 2004, 5, 718-726. [CrossRef] [PubMed]

31. Tao, G.; Cai, R.; Wang, Y.; Song, K.; Guo, P.; Zhao, P.; Zuo, H.; He, H. Biosynthesis and characterization of agnps-silk/PVA film for potential packaging application. Materials 2017, 10, 667. [CrossRef] [PubMed]

32. Cai, R.; Tao, G.; He, H.; Guo, P.; Yang, M.; Ding, C.; Zuo, H.; Wang, L.; Zhao, P.; Wang, Y. In situ synthesis of silver nanoparticles on the polyelectrolyte-coated sericin/pva film for enhanced antibacterial application. Materials 2017, 10, 967. [CrossRef] [PubMed]

33. Jiang, S.; Qin, Y.; Yang, J.; Li, M.; Xiong, L.; Sun, Q. Enhanced antibacterial activity of lysozyme immobilized on chitin nanowhiskers. Food Chem. 2017, 221, 1507-1513. [CrossRef] [PubMed]

34. Chen, C.; Pan, F.; Zhang, S.; Hu, J.; Cao, M.; Wang, J.; Xu, H.; Zhao, X.; Lu, J.R. Antibacterial activities of short designer peptides: A link between propensity for nanostructuring and capacity for membrane destabilization. Biomacromolecules 2010, 11, 402-411. [CrossRef] [PubMed]

35. Wu, Y.-B.; Yu, S.-H.; Mi, F.-L.; Wu, C.-W.; Shyu, S.-S.; Peng, C.-K.; Chao, A.-C. Preparation and characterization on mechanical and antibacterial properties of chitsoan/cellulose blends. Carbohydr. Polym. 2004, 57, 435-440. [CrossRef]

36. Zhou, B.; Li, Y.; Deng, H.; Hu, Y.; Li, B. Antibacterial multilayer films fabricated by layer-by-layer immobilizing lysozyme and gold nanoparticles on nanofibers. Colloids Surf. B 2014, 116, 432-438. [CrossRef] [PubMed]

37. Zhou, T.; Huang, Y.; Li, W.; Cai, Z.; Luo, F.; Yang, C.J.; Chen, X. Facile synthesis of red-emitting lysozyme-stabilized ag nanoclusters. Nanoscale 2012, 4, 5312-5315. [CrossRef] [PubMed]

38. Chuang, C.-C.; Prasannan, A.; Huang, B.-R.; Hong, P.-D.; Chiang, M.-Y. Simple synthesis of eco-friendly multifunctional silk-sericin capped zinc oxide nanorods and their potential for fabrication of hydrogen sensors and UV photodetectors. ACS Sustain. Chem. Eng. 2017, 5, 4002-4010. [CrossRef]

39. Al-Abboodi, A.; Fu, J.; Doran, P.M.; Tan, T.T.Y.; Chan, P.P.Y. Injectable 3D hydrogel scaffold with tailorable porosity post-implantation. Adv. Healthc. Mater. 2014, 3, 725-736. [CrossRef] [PubMed]

40. Annabi, N.; Nichol, J.W.; Zhong, X.; Ji, C.; Koshy, S.; Khademhosseini, A.; Dehghani, F. Controlling the porosity and microarchitecture of hydrogels for tissue engineering. Tissue Eng. Part B 2010, 16, 371-383. [CrossRef] [PubMed] 
41. Zhang, X.; Wyeth, P. Using FTIR spectroscopy to detect sericin on historic silk. Sci. China Chem. 2010, 53, 626-631. [CrossRef]

42. Verma, V.K.; Subbiah, S. Prospects of silk sericin as an adsorbent for removal of ibuprofen from aqueous solution. Ind. Eng. Chem. Res. 2017, 56, 10142-10154. [CrossRef]

43. Singh, Y.P.; Bhardwaj, N.; Mandal, B.B. Potential of agarose/silk fibroin blended hydrogel for in vitro cartilage tissue engineering. ACS Appl. Mater. Inteerfaces 2016, 8, 21236-21249. [CrossRef] [PubMed]

44. Wu, T.; Huang, J.; Jiang, Y.; Hu, Y.; Ye, X.; Liu, D.; Chen, J. Formation of hydrogels based on chitosan/alginate for the delivery of lysozyme and their antibacterial activity. Food Chem. 2018, 240, 361-369. [CrossRef] [PubMed]

45. Gupta, D.; Agrawal, A.; Rangi, A. Extraction and characterization of silk sericin. Indian J. Fibre Text. 2014, 39, 364-372.

46. Tao, G.; Liu, L.; Wang, Y.; Chang, H.; Zhao, P.; Zuo, H.; He, H. Characterization of silver nanoparticlein situsynthesis on porous sericin gel for antibacterial application. J. Nanomater. 2016, 2016, 1-8. [CrossRef]

47. Tao, G.; Wang, Y.; Liu, L.; Chang, H.; Zhao, P.; He, H. Preparation and characterization of silver nanoparticles composited on polyelectrolyte film coated sericin gel for enhanced antibacterial application. Sci. Adv. Mater. 2016, 8, 1547-1552. [CrossRef]

48. Cai, R.; Tao, G.; He, H.; Song, K.; Zuo, H.; Jiang, W.; Wang, Y. One-step synthesis of silver nanoparticles on polydopamine-coated sericin/polyvinyl alcohol composite films for potential antimicrobial applications. Molecules 2017, 22, 721. [CrossRef] [PubMed]

49. Cao, T.T.; Zhang, Y.Q. Processing and characterization of silk sericin from bombyx mori and its application in biomaterials and biomedicines. Mater. Sci. Eng. C 2016, 61, 940-952. [CrossRef] [PubMed]

50. Silva, V.R.; Hamerski, F.; Weschenfelder, T.A.; Ribani, M.; Gimenes, M.L.; Scheer, A.P. Equilibrium, kinetic, and thermodynamic studies on the biosorption of bordeaux s dye by sericin powder derived from cocoons of the silkwormbombyx mori. Desalin. Water Treat. 2015, 57, 5119-5129. [CrossRef]

51. Shemesh, M.; Zilberman, M. Structure-property effects of novel bioresorbable hybrid structures with controlled release of analgesic drugs for wound healing applications. Acta Biomater. 2014, 10, 1380-1391. [CrossRef] [PubMed]

52. Atar-Froyman, L.; Sharon, A.; Weiss, E.I.; Houri-Haddad, Y.; Kesler-Shvero, D.; Domb, A.J.; Pilo, R.; Beyth, N. Anti-biofilm properties of wound dressing incorporating nonrelease polycationic antimicrobials. Biomaterials 2015, 46, 141-148. [CrossRef] [PubMed]

53. Liu, Y.; Sun, Y.; Xu, Y.; Feng, H.; Fu, S.; Tang, J.; Liu, W.; Sun, D.; Jiang, H.; Xu, S. Preparation and evaluation of lysozyme-loaded nanoparticles coated with poly-gamma-glutamic acid and chitosan. Int. J. Biol. Macromol. 2013, 59, 201-207. [CrossRef] [PubMed]

54. Ibrahim, H.R.; Higashiguchi, S.; Juneja, L.R.; Kim, M.; Yamamoto, T. A structural phase of heat-denatured lysozyme with novel antimicrobial action. J. Agric. Food Chem. 1996, 44, 1416-1423. [CrossRef]

55. Bernkop-Schnürch, A.; Krist, S.; Vehabovic, M.; Valenta, C. Synthesis and evaluation of lysozyme derivatives exhibiting an enhanced antimicrobial action. Eur. J. Pharm. Sci. 1998, 6, 301-306. [CrossRef]

56. Wu, T.; Wu, C.; Fu, S.; Wang, L.; Yuan, C.; Chen, S.; Hu, Y. Integration of lysozyme into chitosan nanoparticles for improving antibacterial activity. Carbohydr. Polym. 2017, 155, 192-200. [CrossRef] [PubMed]

57. Yu, K.; Lu, F.; Li, Q.; Chen, H.; Lu, B.; Liu, J.; Li, Z.; Dai, F.; Wu, D.; Lan, G. In situ assembly of ag nanoparticles (agnps) on porous silkworm cocoon-based would film: Enhanced antimicrobial and wound healing activity. Sci. Rep. 2017, 7, 2107. [CrossRef] [PubMed]

58. Kamoun, E.A.; Kenawy, E.-R.S.; Tamer, T.M.; El-Meligy, M.A.; Mohy Eldin, M.S. Poly (vinyl alcohol)-alginate physically crosslinked hydrogel membranes for wound dressing applications: Characterization and bio-evaluation. Arab. J. Chem. 2015, 8, 38-47. [CrossRef]

59. Yao, Y.; Liu, H.; Ding, X.; Jing, X.; Gong, X.; Zhou, G.; Fan, Y. Preparation and characterization of silk fibroin/poly(L-lactide-co- $\varepsilon$-caprolactone) nanofibrous membranes for tissue engineering applications. J. Bioact. Compat. Polym. 2015, 30, 633-648. [CrossRef]

(C) 2018 by the authors. Licensee MDPI, Basel, Switzerland. This article is an open access article distributed under the terms and conditions of the Creative Commons Attribution (CC BY) license (http:// creativecommons.org/licenses/by/4.0/). 